\title{
Patient Blood Management in Craniosynostosis Surgery
}

\section{Claudine Kumba}

Department of Pediatric and Obstetric Anesthesia and Critical Care, Hôpital Universitaire Necker Enfants Malades, Assistance Publique Hôpitaux de Paris, APHP, Université de Paris, Paris, France

Email: claudine.kumba@gmail.com

How to cite this paper: Kumba, C. (2021) Patient Blood Management in Craniosynostosis Surgery. Open Journal of Modern Neurosurgery, 11, 211-222. https://doi.org/10.4236/ojmn.2021.114025

Received: August 10, 2021

Accepted: September 7, 2021

Published: September 10, 2021

Copyright $\odot 2021$ by author(s) and Scientific Research Publishing Inc. This work is licensed under the Creative Commons Attribution International License (CC BY 4.0).

http://creativecommons.org/licenses/by/4.0/ (c) (i) Open Access

\begin{abstract}
Background: Craniosynostosis surgery is one of the most hemorrhagic interventions, where transfusion rates vary from $20 \%$ to $100 \%$ depending on the study. Objective: To describe intraoperative and postoperative outcomes in a secondary analysis of children who underwent craniosynostosis surgery included in the initial retrospective study with the aim of proposing intraoperative implementation optimization protocols for postoperative outcome improvement. Methods: Secondary analysis. The study was approved by the Ethics Committee. Results: There were 69 children with a median age of 10 [0 - 207] months. Eight (11.6\%) patients had intraoperative and/or postoperative complications. One patient $(1.5 \%)$ had intraoperative hemorrhagic shock, and two patients (2.9\%) had intraoperative broncholaryngospasm. One patient (1.5\%) had postoperative anaphylaxis. One patient (1.5\%) had postoperative hemorrhagic shock. One patient $(1.5 \%)$ had postoperative respiratory failure. Two patients $(2.9 \%)$ had postoperative neurologic failure. One patient (1.5\%) had neuro-meningeal sepsis. One patient (1.5\%) had a re-operation. There was no in-hospital mortality. Fourty-eight patients (69.6\%) had intraoperative transfusions. Conclusion: Transfusion protocols guided with point-of-care tests should be included in patient blood management programs in craniosynostosis surgery.
\end{abstract}

\section{Keywords}

Craniosynostosis, Children, Transfusion, Outcome, Point of Care Viscoelastic Assays, Rotational Thromboelastometry

\section{Introduction}

Cranial vault remodeling surgery is one of the most hemorrhagic interventions, where transfusion rates vary from $20 \%$ to $100 \%$ depending on the study [1] [2] 
[3]. Depending on the type of craniosynostosis, intraoperative blood loss has been reported to be higher in syndromic cranial vault remodeling, where venous anomalies play a major role in this outcome [4] [5]. Tranexamic acid and higher fibrinogen levels have demonstrated a reduction in transfusion requirements and blood loss in craniosynostosis surgery [6] [7].

It has been reported that transfusion with all types of blood products was one of the independent predictors of adverse postoperative outcome in terms of morbidity and length of hospital stay [8] [9]. Hospitalization costs were increased in transfused patients compared with non-transfused patients [10]. Preoperative, intraoperative and postoperative hemoglobin levels have been correlated with postoperative morbidity, length of hospital stay and length of mechanical ventilation in surgical pediatric patients [11]. Transfusion can be a necessary therapeutic intervention in hemorrhagic settings, and it is important to assess the benefits and risks when deciding to administer blood products in patients. Several physio-pathologic mechanisms underlie adverse outcomes correlated with transfusion [12]. First, transfusion can be related to infectious risks due to transfusion-transmitted viruses, bacterial contamination, vector-borne bacteria, parasites and prions [12]. Second, transfusion can be related to immunological risks that can lead to transfusion-related acute injury known as TRALI; immunological risks can lead to febrile nonhemolytic transfusion reactions, allergic and anaphylactic reactions, hemolytic transfusion reactions, transfusion-related immunomodulation known as TRIM, posttransfusion purpura and transfusion-associated graft versus host disease [12].

Finally, transfusion can be related to noninfectious and nonimmunological risks, which can be due to mis-transfusion and can be expressed as transfusion-associated circulatory overload known as TACO and as coagulopathy complications in massive transfusion [12].

The incidence of transfusion-related acute lung injury in critically ill patients has been reported to be $6.9 \%$. Reported predictors of TRALI were mechanical ventilation, sepsis and high risk of mortality ill score [13]. The hypothesis underlying TRALI is the presence of antibodies or other inflammation mediators in blood products [13].

Transfusion-related immunomodulation is probably due to complex reactions that lead to dual proinflammatory and immunosuppressive responses [14].

Transfused patients are generally critically ill, and critical illness is associated with acute inflammation and immunosuppression.

We described here intraoperative and postoperative outcomes in a secondary analysis of patients who underwent craniosynostosis surgery included in the initial retrospective study [9]. This outcome description in this potential hemorrhagic setting had the objectives of proposing intraoperative implementation optimization protocols for postoperative outcome improvement.

\section{Methods and Materials}

A secondary analysis of patients who underwent cranial vault remodeling was 
performed in the initial retrospective study [9].

The study was approved by the Ethics Committee of Necker Enfants Malades University Hospital under registration number 2017-CK-5-R1 on 21 March 2017.

Patients were retrospectively included from 1 January 2014 to 17 May 2017.

The inclusion criteria were all children included in the initial study who underwent craniosynostosis surgery and were less than 18 years old (see Figure 1, inclusion and exclusion criteria flow chart).

The exclusion criteria were patients included in the initial study who did not undergo craniosynostosis surgery and were aged more than 18 years old (see Figure 1).

Outcomes included intraoperative and postoperative organ dysfunction, length of hospital stay (LOS), length of intensive care unit stay (LOSICU), total length of hospital stay (TLOS = LOS + LOSICU), length of mechanical ventilation (LMV), and intraoperative blood product requirements.

Organ dysfunction was defined as a state of organ alteration with clinical, laboratory and imaging findings including infection that was not present in the preoperative period or that was present preoperatively with postoperative majoration or increase.

Patients were followed until discharge from hospital.

Statistics were analyzed with XLSTAT 2020.4.1. software. Continuous variables were expressed as medians with ranges or means with standard deviations. Categorial variables were described in proportions.

In our hospital, patients who underwent craniosynostosis surgery were managed intraoperatively according to a defined protocol. All patients were monitored with an arterial and central venous catheter, an indwelling bladder catheter, nasogastric tubing, a high-volume fluid administrating device, a Bair Hugger ${ }^{\circledast}$, and a core temperature probe. Induction of anesthesia was performed with sevoflurane, sufentanil at a $0.3-0.5 \mu \mathrm{g} / \mathrm{kg}$ bolus intravenously (IV) and with a short-acting nondepolarizing muscle relaxant such atracurium at a $0.5 \mathrm{mg} / \mathrm{kg}$ bolus IV. Airway was secured with oro-tracheal intubation. Antibiotic prophylaxis

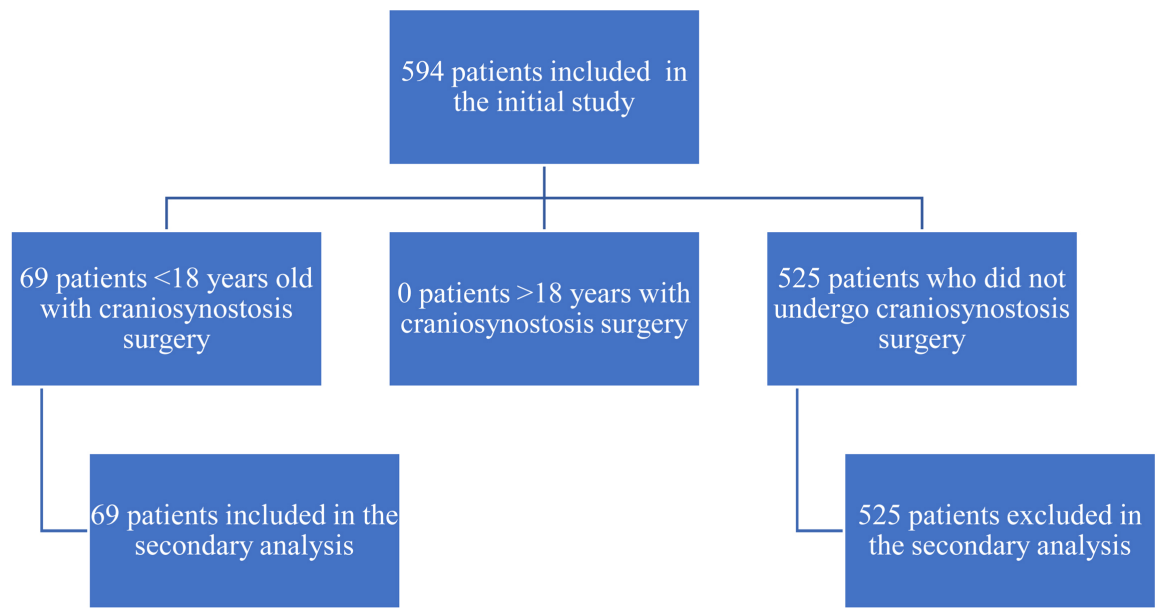

Figure 1. Inclusion and exclusion criteria flow chart. 
was performed with cefazolin at a $50 \mathrm{mg} / \mathrm{kg}$ bolus IV. Maintenance of anesthesia was realized with sufentanil as an IV infusion of $0.3-0.5 \mu \mathrm{g} / \mathrm{kg} / \mathrm{h}$ with sevoflurane. All patients received tranexamic acid as a $10 \mathrm{mg} / \mathrm{kg}$ IV bolus followed by an IV infusion of $10 \mathrm{mg} / \mathrm{kg} / \mathrm{h}$ up to 6 hours postoperatively. A maintenance infusion was performed with a crystalloid (isopedia ${ }^{\oplus}$ ) at $3 \mathrm{ml} / \mathrm{kg} / \mathrm{h}$ if the patient weighed less than $10 \mathrm{~kg}$ and $5 \mathrm{ml} / \mathrm{kg} / \mathrm{h}$ if the weight was above $10 \mathrm{~kg}$. Hemoglobin levels were assessed on a regular basis. Fluid therapy was managed by monitoring central venous pressure, arterial blood pressure and pulse pressure variation with colloids (voluven ${ }^{\circledast}$ or plasmion ${ }^{\circledR}$ ) administered as a $20-30 \mathrm{ml} / \mathrm{kg}$ bolus, packed red blood cells (PRBCs) and albumin. Fresh frozen plasma (FFP) at 15 $\mathrm{ml} / \mathrm{kg}$ and concentrated platelet units at $0.1-0.2 \mathrm{UI} / \mathrm{kg}$ (CUP) were administered if transfusion requirements were above the total circulating blood volume. The volume of transfused packed red blood cells was determined as followed: $\mathrm{PRBC}$ in $\mathrm{ml}=$ Target hematocrit levels-Initial hematocrit levels/Weight in $\mathrm{kg}$.

After surgery, the patient was transferred sedated and intubated in the post-anesthesia care unit (PACU) or the pediatric intensive care unit (PICU) for surveillance.

Postoperative analgesia was realized with intravenous morphine and oral morphine, intravenous acetaminophen and intrarectal ibuprofen. Patients could start oral intake feeding 4 hours after extubation. Blood draining systems were removed on postoperative day 2 . All patients received intravenously $5 \mathrm{mg} / \mathrm{kg}$ iron (venofer ${ }^{\circledR}$ ) as an infusion. Patients were discharged from the PICU when hemoglobin levels equaled or were above $12 \mathrm{~g} / \mathrm{dL}$.

\section{Results}

Table 1 illustrates the general characteristics. For more details see Figure S1 in Supplemental files.

There were 69 children with a median age of 10 [0 - 207] months. There were thirty-six (52.2\%), sixteen (23.2\%), fourteen (20.3\%) and three (4.3\%) American Society of Anesthesiologists grade one, two, three and four patients, respectively. Four (5.8\%) patients had an emergency intervention, and sixty-five (94.2\%) had an elective surgery. Eight (11.6\%) patients had intraoperative and/or postoperative complications. One patient $(1.5 \%)$ had intraoperative hemorrhagic shock, and two patients (2.9\%) had intraoperative broncholaryngospasm. One patient (1.5\%) had postoperative anaphylaxis. One patient (1.5\%) had postoperative hemorrhagic shock. One patient (1.5\%) had postoperative respiratory failure. Two patients $(2.9 \%)$ had postoperative neurologic failure. One patient (1.5\%) had neuro-meningeal sepsis. One patient (1.5\%) had a re-operation.

There was no in-hospital mortality.

Fourty-eight patients (69.6\%) had intraoperative transfusion with PRBC, FFP or CUP.

Median PRBC volume was $1[0-4]$ units. The median FFP volume was 0 [0 2] units, and the median CUP volume was 0 [0 - 2] units. The mean preoperative 
Table 1. General characteristics.

\begin{tabular}{|c|c|}
\hline Characteristic & $\mathrm{N}=69$ \\
\hline Median age months [range] & $10[0-207]$ \\
\hline ASA I n (\%) & $36(52.2)$ \\
\hline ASA II n (\%) & $16(23.2)$ \\
\hline ASA III n (\%) & $14(20.3)$ \\
\hline ASA IV n (\%) & $3(4.3)$ \\
\hline Emergency surgery n (\%) & $4(5.8)$ \\
\hline Elective surgery n (\%) & $65(94.2)$ \\
\hline Re-operation n (\%) & $1(1.5)$ \\
\hline $\begin{array}{l}\text { Patients with intra-operative and or postoperative complications } \\
\text { (organ failure or sepsis) } n(\%)\end{array}$ & $8(11.6)$ \\
\hline Intra-operative hemorrhagic shock n (\%) & $1(1.5)$ \\
\hline Intra-operative broncho-laryngospasm n (\%) & $2(2.9)$ \\
\hline Postoperative anaphylaxis n (\%) & $1(1.5)$ \\
\hline Postoperative hemorrhagic shock n (\%) & $1(1.5)$ \\
\hline Postoperative respiratory failure $\mathrm{n}(\%)$ & $1(1.5)$ \\
\hline Postoperative neurologic failure n (\%) & $2(2.9)$ \\
\hline Postoperative neuro-meningeal sepsis n (\%) & $1(1.5)$ \\
\hline In-hospital mortality n (\%) & $0(0)$ \\
\hline Transfusion n (\%) & $48(69.6)$ \\
\hline Median packed red blood cells units [range] & $1[0-4]$ \\
\hline Median fresh frozen plasma volume units [range] & $0[0-2]$ \\
\hline Median concentrated platelet units [range] & $0[0-2]$ \\
\hline Mean preoperative hemoglobin levels \pm standard deviation in $\mathrm{g} / \mathrm{dL}$ & $12.0 \pm 1.2$ \\
\hline Mean postoperative hemoglobin levels \pm standard deviation in $\mathrm{g} / \mathrm{dL}$ & $11.6 \pm 1.6$ \\
\hline Median crystalloid volume in $\mathrm{ml}$ [range] & $0[0-1000]$ \\
\hline Median colloid volume in $\mathrm{ml}$ [range] & $250[30-2050]$ \\
\hline Median length of intensive care unit stay in days [range] & $3[1-90]$ \\
\hline Median length of hospital stay in days [range] & $2[0-8]$ \\
\hline Median total length of hospital stay in days [range] & $5[2-90]$ \\
\hline Median total length of mechanical ventilation in days [range] & $0[0-79]$ \\
\hline
\end{tabular}

hemoglobin level was $12.0 \pm 1.2 \mathrm{~g} / \mathrm{dL}$, and the mean postoperative hemoglobin level was $11.6 \pm 1.6 \mathrm{~g} / \mathrm{dL}$.

Median crystalloid volume was 0 [0 - 1000] ml. Median colloid volume was 250 [30 - 2050] ml.

The median length of intensive care unit stay (LOSICU) was 3 [1 - 90] days. The median length of hospital stay (LOS) was $2[0-8]$ days. The median total length of hospital stay (LOSICU + LOS = TLOS) was 5 [2 - 90] days. The median length of mechanical ventilation (LMV) was 0 [0 - 79] days.

Table 2 illustrates co-morbidities. 
Table 2. Co-morbidities.

\begin{tabular}{|c|c|}
\hline Co-morbidity & $\begin{array}{l}\text { Number of patients } \\
\text { (\%) }\end{array}$ \\
\hline Saethre-Chotzen syndrome & $1(1.5)$ \\
\hline $\begin{array}{l}\text { Polymal formation syndrome with congenital heart disease and } \\
\text { metabolic disease }\end{array}$ & $1(1.5)$ \\
\hline Apert syndrome & $2(2.9)$ \\
\hline Arachnoid cyst & $1(1.5)$ \\
\hline Asthma & $2(2.9)$ \\
\hline Chiari's malformation & $2(2.9)$ \\
\hline Congenital coagulation disorder & $1(1.5)$ \\
\hline Congenital heart disease & $2(2.9)$ \\
\hline Crouzon syndrome & $6(8.7)$ \\
\hline Epilepsy & $1(1.5)$ \\
\hline Former pre-term & $2(2.9)$ \\
\hline Intracerebral tumor & $1(1.5)$ \\
\hline Obstructive apneic syndrome & $3(4.3)$ \\
\hline Rachitism & $1(1.5)$ \\
\hline Tracheomalacia & $1(1.5)$ \\
\hline
\end{tabular}

The most common comorbidity was Crouzon syndrome in six patients (8.7\%), followed by obstructive apneic syndrome in three patients (4.3\%), Apert syndrome, asthma, Chiari's malformation, congenital heart disease, and former preterm birth in two patients (2.9\%).

\section{Discussion}

The intraoperative transfusion rate in this secondary cohort was $69.6 \%$. Most of the patients received PRBC and/or FFP units. Cranial vault modeling is a potential hemorrhagic surgery, and intraoperative transfusion with point-of-care viscoelastic assays needs to be included in patients who undergo this intervention. It has been proven that point-of-care tests to guide transfusion in hemorrhagic surgery, such as craniosynostosis, reduce transfusion requirements [6] [15] [16]. Figures 2-4 illustrate algorithms with rotational thromboelastometry (ROTEM) in different age groups. ROTEM parameters in children have been described with ROTEM delta version [17]. These algorithms can be applied in other potential hemorrhagic settings to guide blood product administration [18] [19]. In this secondary cohort, the intraoperative hemorrhagic shock rate was $1.5 \%$. Syndromic craniosynostosis has been reported to be correlated with higher intraoperative blood loss, emphasizing the necessity of integrating point-of-care tests for transfusion optimization in these patients [4]. Cranial vault surgery concerns small infants most of the time, and blood loss in these patients can occur acutely and rapidly; thus, anticipating this situation is mandatory. The rates of intraoperative hemorrhagic shock in children vary according to surgical 


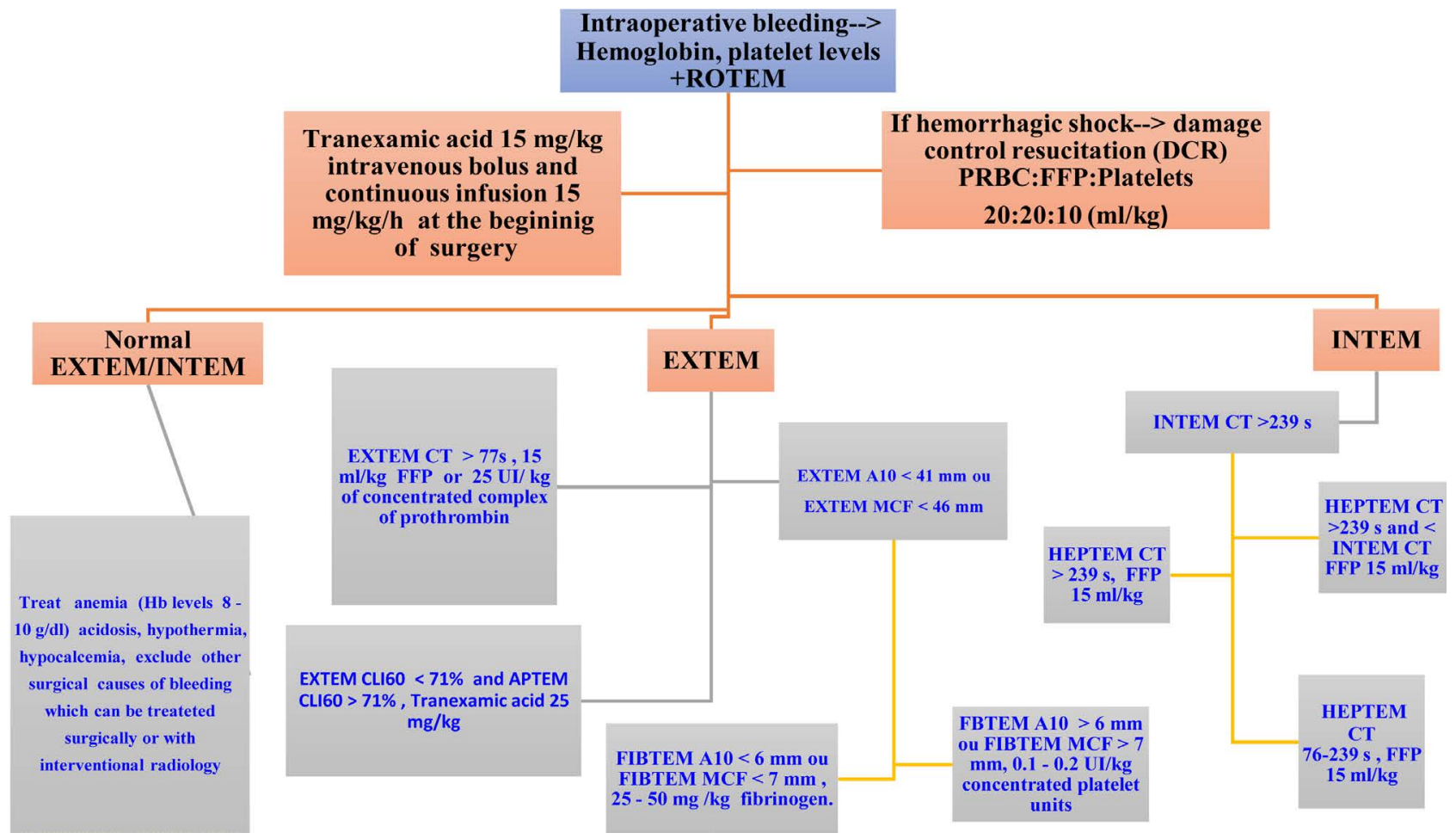

Figure 2. ROTEM algorithm in children between enfant $0-24$ months. CT $=$ coagulation time in seconds, A10 = clot firmness at 10 minutes, $\mathrm{MCF}=$ maximum clot firmness, CLI60 = lysis index in \% 60 minutes after CT, ML = maximum lysis in \%, $\mathrm{FFP}=$ fresh frozen plasma, $\mathrm{PRBC}=$ packed red blood cells, $\mathrm{Hb}=$ hemoglobin.

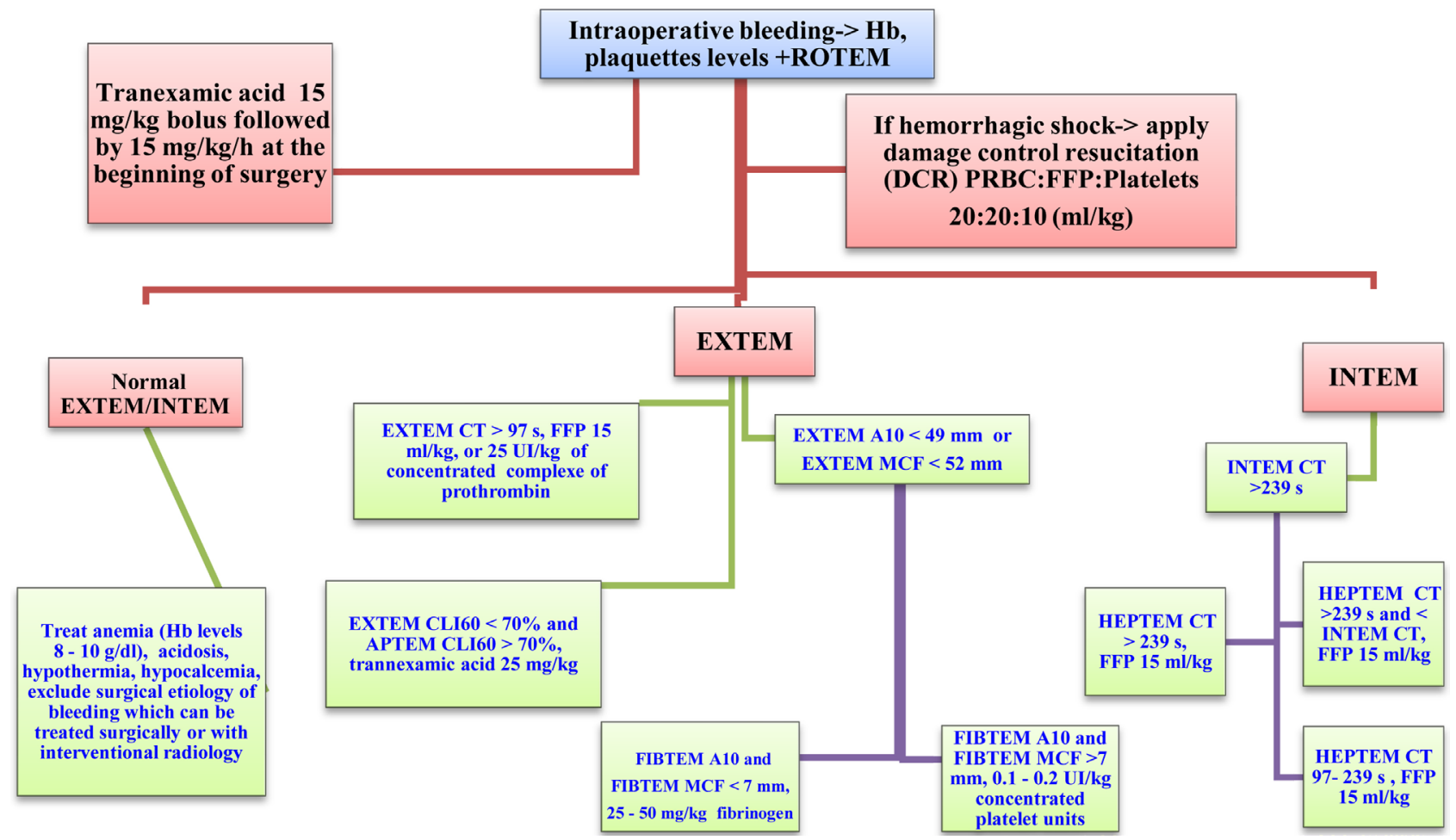

Figure 3. ROTEM algorithm in children $2-16$ years. CT = coagulation time in seconds, A10 = clot firmness after 10 minutes, $\mathrm{MCF}=$ maximum clot firmness, CLI60 = lysis index in \% 60 minutes after CT, ML = maximum lysis in \%, FFP = fresh frozen plasma, $\mathrm{PRBC}=$ packed red blood cells, $\mathrm{Hb}=$ hemoglobin. 


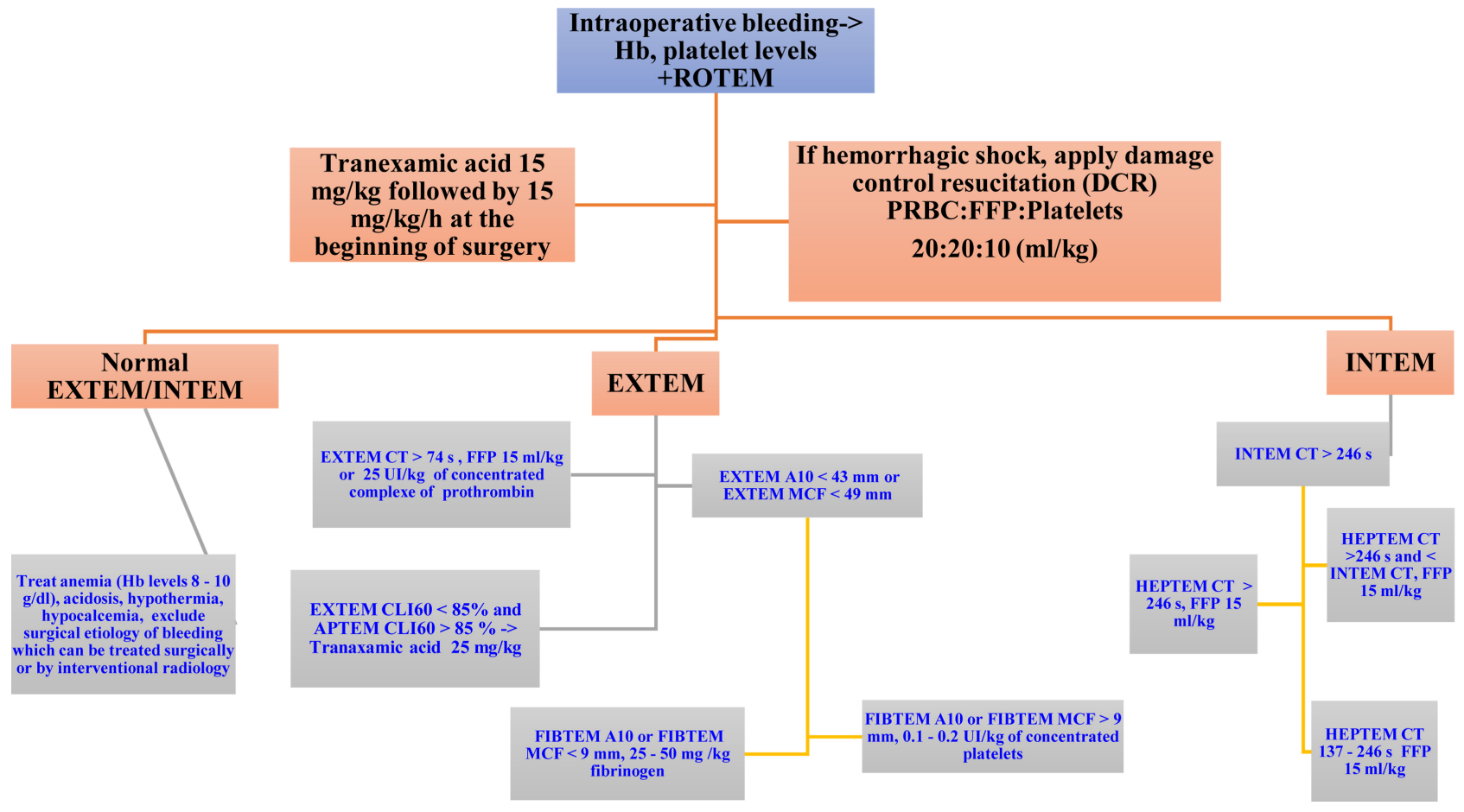

Figure 4. ROTEM Algorithm $>16$ years. $\mathrm{CT}=$ coagulation time in seconds, $\mathrm{A} 10=$ clot firmness at 10 minutes, $\mathrm{MCF}=$ maximum clot firmness, CLI60 = lysis index in \% 60 minutes after CT, ML = maximum lysis in \%, FFP = fresh frozen plasma, $\mathrm{PRBC}=$ packed red blood cells, $\mathrm{Hb}=$ hemoglobin.

settings and age and have been reported to vary between 0 and 5.6\% [20]-[25]. In small children, blood product requirements can rapidly reach total circulating blood volume, and massive transfusion is very likely in potential hemorrhagic situations. Transfusion and massive blood product administration are predictors of adverse postoperative outcomes that must be put into balance with the risk of anemia, which is also a predictor of postoperative unfavorable evolution in surgical children [10] [11] [12] [13] [14] [25] [26] [27] [28]. Preoperative and intraoperative hemoglobin levels have been negatively correlated with postoperative outcome; hemoglobin levels below $6 \mathrm{~g} / \mathrm{dL}$ and intraoperative hemoglobin levels below $5 \mathrm{~g} / \mathrm{dL}$ have been related to higher LOS [11]. Postoperative hemoglobin levels have been positively correlated with LMV, with hemoglobin levels higher than $12 \mathrm{~g} / \mathrm{dL}$ being related to higher LMV. Restrictive transfusion strategies compared to liberal strategies in critically ill patients did not increase adverse outcomes. Maintaining a hemoglobin level target of $12 \mathrm{~g} / \mathrm{dL}$ or more is not necessary for all patients and should be assessed according to patients global status and co-existing co-morbidities like congenital heart disease, prematurity, sepsis etc.

Preoperative erythropoietin has been reported to reduce blood product transfusion in craniosynostosis surgery, and integrating this molecule with iron supplementation in blood transfusion management protocols in this surgery could contribute to reducing the rate of intraoperative transfusions [29].

Crystalloid and colloid fluid therapy, similar to transfusion, need to be guided 
with validated variables and tools in the pediatric population for optimal fluid and hemodynamic management [30] [31] [32] [33]. Non-optimal intraoperative values of cerebral and renal regional oxygen saturation assessed with near-infrared spectroscopy (NIRS), mixed venous oxygen saturation and lactate levels were predictors of adverse postoperative outcome in terms of organ dysfunction and LOS. Monitoring these parameters intraoperatively and optimizing them intraoperatively could improve postoperative outcome in major surgical settings [33]. Intraoperative fluid therapy optimization with validated tools in children could also help improve postoperative outcome in this surgery [32].

\section{Conclusion}

Transfusion protocols guided with point-of-care tests should be included in patient blood management programs in craniosynostosis surgery. Targeting higher postoperative hemoglobin levels in all patients is not necessary and should be assessed depending on patient comorbidities. Restrictive transfusion strategies are alternatives to liberal practices to reduce transfusion rates. Intraoperative operative fluid optimization with validated tools in children could also help to improve postoperative outcome in this surgery.

\section{Presentation of Preliminary Data}

This manuscript has been registered as a preprint on the preprint platform Research Square under the DOI registration number https://doi.org/10.21203/rs.3.rs-774234/v1.

\section{Author Contributions}

Claudine Kumba conceptualized and designed the study and drafted the initial manuscript. She designed the data collection instruments, collected data, carried out initial and final analyses.

\section{Ethics Approval}

This study received approval from the Ethics Committee of Necker on 21 March 2017 under registration number 2017-CK-5-R1 and waived patient consent.

\section{Conflicts of Interest}

The author declared no conflicts of interest.

\section{References}

[1] Bonfield, C.M., Sharma, J., Cochrane, D.D., et al. (2016) Minimizing Blood Transfusions in the Surgical Correction of Craniosynostosis: A 10-Year Single Center Experience. Child's Nervous System, 32, 143-151. https://doi.org/10.1007/s00381-015-2900-6

[2] Beethe, A.B., Spitznagel, R.A., Kugler, J.A., Goeller, J.K., et al. (2020) The Road to Tranfusion-Free Craniosynostosis Repair in Children Less than 24 Months Old: A Quality Improvement Initiative. Pediatric Quality \& Safety, 4, e331. 
https://doi.org/10.1097/pq9.0000000000000331

[3] Albright, A.L., Pollack, I.F., Adelson, P.D. and Solot, J.J. (1999) Outcome Data and Analysis in Pediatric Neurosurgery. Neurosurgery, 45, 101-106. https://doi.org/10.1097/00006123-199907000-00025

[4] Copeland, A.E., Hoffman, C.E., Tsitouras, V., et al. (2018) Clinical Significance of Venous Anomalies in Syndromic Craniosynostosis. Plastic and Reconstructive Surgery_Global Open, 6, e1613. https://doi.org/10.1097/GOX.0000000000001613

[5] Bartz-Kurycki, M., Wei, S., Bernardi, K., et al. (2019) Impact of Cardiac Risk Factors on Complications Following Cranial Vault Remodeling: Analysis of the 2012-2016 NSQIP-P Database. Journal of Craniofacial Surgery, 30, 442-447. https://doi.org/10.1097/SCS.0000000000005114

[6] Haas, T., Spielmann, N., Restin, T., et al. (2016) Economic Aspects of Intraoperative Coagulation Management Targeting Higher Fibrinogen Concentrations during Major Craniosynostosis Surgery. Pediatric Anesthesia, 26, 77-83. https://doi.org/10.1111/pan.12784

[7] Faraoni, D. and Goobie, S.M. (2014) The Efficacy of Antfibrinolytic Drugs in Children Undergoing Noncardiac Surgery: A Systematic Review of the Literature. Anesthesia \& Analgesia, 118, 628-636. https://doi.org/10.1213/ANE.0000000000000080

[8] Kumba, C., Taright, H., Terzi, E., Telion, C., Beccaria, K., Paternoster, G., Zerah, M., Bustarret, O., Jugie, M., Rubinsztajn, R. and Treluyer, J.M. (2018) Blood Product Transfusion and Postoperative Outcome in Pediatric Neurosurgical Patients. EC Anaesthesia, 4, 288-298.

[9] Kumba, C., Cresci, F., Picard, C., et al. (2017) Transfusion and Morbi-Mortality Factors: An Observational Descriptive Retrospective Pediatric Cohort Study. Journal of Anesthesia \& Critical Care: Open Access, 8, Article No. 00315. https://doi.org/10.15406/jaccoa.2017.08.00315

[10] Kumba, C. (2021) Transfusion in Pediatric Surgical Settings: Economic Aspects and Hospitalization Costs. Acta Scientific Paediatrics, 4, 3-6.

https://doi.org/10.31080/ASPE.2021.04.0390

[11] Kumba, C. (2021) Hemoglobin Levels and Postoperative Outcome in Pediatric Surgical Patients. https://doi.org/10.21203/rs.3.rs-669069/v1

[12] El Kenz, H. and Van der Linden, P. (2013) Transfusion-Related Acute Lung Injury. European Journal of Anaesthesiology, 31, 345-350.

[13] Mulder, H.D., Augustijn, Q.J., Van Woensel, J.B., et al. (2015) Incidence, Risk Factors, and Outcome of Transfusion-Related Acute Lung Injury in Critically Ill Children: A Retrospective Study. Journal of Critical Care, 30, 55-59.

https://doi.org/10.1016/j.jcrc.2014.10.005

[14] Muszynski, J.A., Spinella, P.C., Cholette, J.M., et al. (2017) Transfusion-Related Immunomodulation: Review of the Literature and Implications for Pediatric Critical Illness. Transfusion, 57, 195-206.

[15] Kumba, C. and Treluyer, J. (2020) Perspectives and Evolution of Intraoperative Transfusion Goal Directed Protocols with Viscoelastic Methods and Perioperative Outcomes in Children. Research in Pediatrics \& Neonatology, 4, RPN.000582.2020. https://doi.org/10.31031/RPN.2020.04.000582

[16] Kumba, C., Querciagrossa, S., Harte, C., Willems, A., et al. (2019) A Systematic Review and Meta-Analysis of Goal Directed Intra-Operative Transfusion Protocols Guided by Viscoelastic Methods and Perioperative Outcomes inChildren. International Journal of Recent Scientific Research, 10, 31466-31471. 
[17] Oswald, E., Stalzer, B., Heitz, E., Weiss, M., Schmugge, Strasak, A., Innerhofer, P. and Haas, T. (2010) Thromboelastometry (ROTEM) in Children: Age-Related Reference Ranges and Correlations with Standard Coagulation Tests. British Journal of Anaesthesia, 105, 827-835. https://doi.org/10.1093/bja/aeq258

[18] Kumba, C. (2021) Liver Transplantation in Children and Impact of Intraoperative Goal-Directed Therapies on Postoperative Outcome.

https://doi.org/10.21203/rs.3.rs-744584/v1

[19] Kumba, C. (2021) Scoliosis in Children: Impact of Goal Directed Therapies on Intraoperative and Postoperative Outcomes. https://doi.org/10.21203/rs.3.rs-765785/v1

[20] Kumba, C. (2021) Preterm Infants in Major Abdominal Surgery and Postoperative Outcome. https://doi.org/10.21203/rs.3.rs-669064/v1

[21] Kumba, C. (2021) Postoperative Outcome in Non-Preterm Infants under One year Old in Non-Cardiac Surgery. Acta Scientific Paediatrics, 4, 11-23. https://doi.org/10.21203/rs.3.rs-638904/v1

[22] Kumba, C. (2021) Children Aged between 1 and 3 Years in Noncardiac Surgery and Postoperative Outcome. EC Paediatrics, 10, 67-74.

[23] Kumba, C. (2021) Postoperative Outcome in Children Aged between 3 and 6 Years in Abdominal Surgery, Neurosurgery and Orthopedics. Pediatric Anesthesia and Critical Care Journal, 9, 43-47.

[24] Kumba, C. (2021) Postoperative Outcome in Children aged between 6 and 10 Years in Major Abdominal Surgery, Neurosurgery and Orthopedic Surgery.

https://doi.org/10.21203/rs.3.rs-669076/v1

[25] Kumba, C. (2021) Major Abdominal Surgery, Neurosurgery, Orthopedic Surgery in Children Aged between 10 and 18 Years and Postoperative Outcome. https://doi.org/10.21203/rs.3.rs-666955/v1

[26] Park, C., Wormald, J., Miranda, B.H., et al. (2017) Perioperative Blood Loss and Transfusion in Craniosynostosis Surgery. Journal of Craniofacial Surgery, 29, 112-115.

[27] Kumba, C. (2019) Iron Deficiency with or without Anemia and Perspectives of Perioperative Management in Children. Advances in Pediatric Research, 6, 33. https://doi.org/10.35248/2385-4529.19.6.33

[28] Faraoni, D. and Goobie, S. (2016) Relationship between Preoperative Anemia and In-Hospital Mortality in Children Undergoing Noncardiac Surgery. Anesthesia \& Analgesia, 123, 1582-1587. https://doi.org/10.1213/ANE.0000000000001499

[29] Aljaaly, H.A., Aldekhayel, S.A., Diaz-Abele, J., et al. (2017) Effect of Erythropoietin on Transfusion Requirements for Craniosynostosis Surgery in Children. Journal of Craniofacial Surgery, 28, 1315-1319. https://doi.org/10.1097/SCS.0000000000003717

[30] Kumba, C. (2020) Physiology Principles Underlying Goal Directed Therapies in Children. Research in Pediatrics \& Neonatology, 4, RPN.000591.2020. https://doi.org/10.31031/RPN.2020.04.000591

[31] Kumba, C. (2020) Rationale of Goal Directed Therapies in Children. Advances in Pediatric Research, 7, 42.

[32] Kumba, C. (2020) Goal Directed Fluid and Hemodynamic Therapy and Postoperative Outcomes in Children: Value of Transthoracic Echocardiographic Aortic Blood Flow Peak Velocity Variation: A Multi-Centre Randomized Controlled Trial Protocol. Advances in Pediatric Research, 7, 35.

https://doi.org/10.35248/2385-4529.20.7.35 
[33] Kumba, C., Willems, A., Querciagrossa, S., et al. (2019) A Systematic Review and Meta-Analysis of Intraoperative Goal Directed Fluid and Haemodynamic Therapy in Children and Postoperative Outcome. Journal of Emergency Medicine \& Critical Care, 5, 9. https://doi.org/10.13188/2469-4045.1000020.

\section{Supplemental Files}

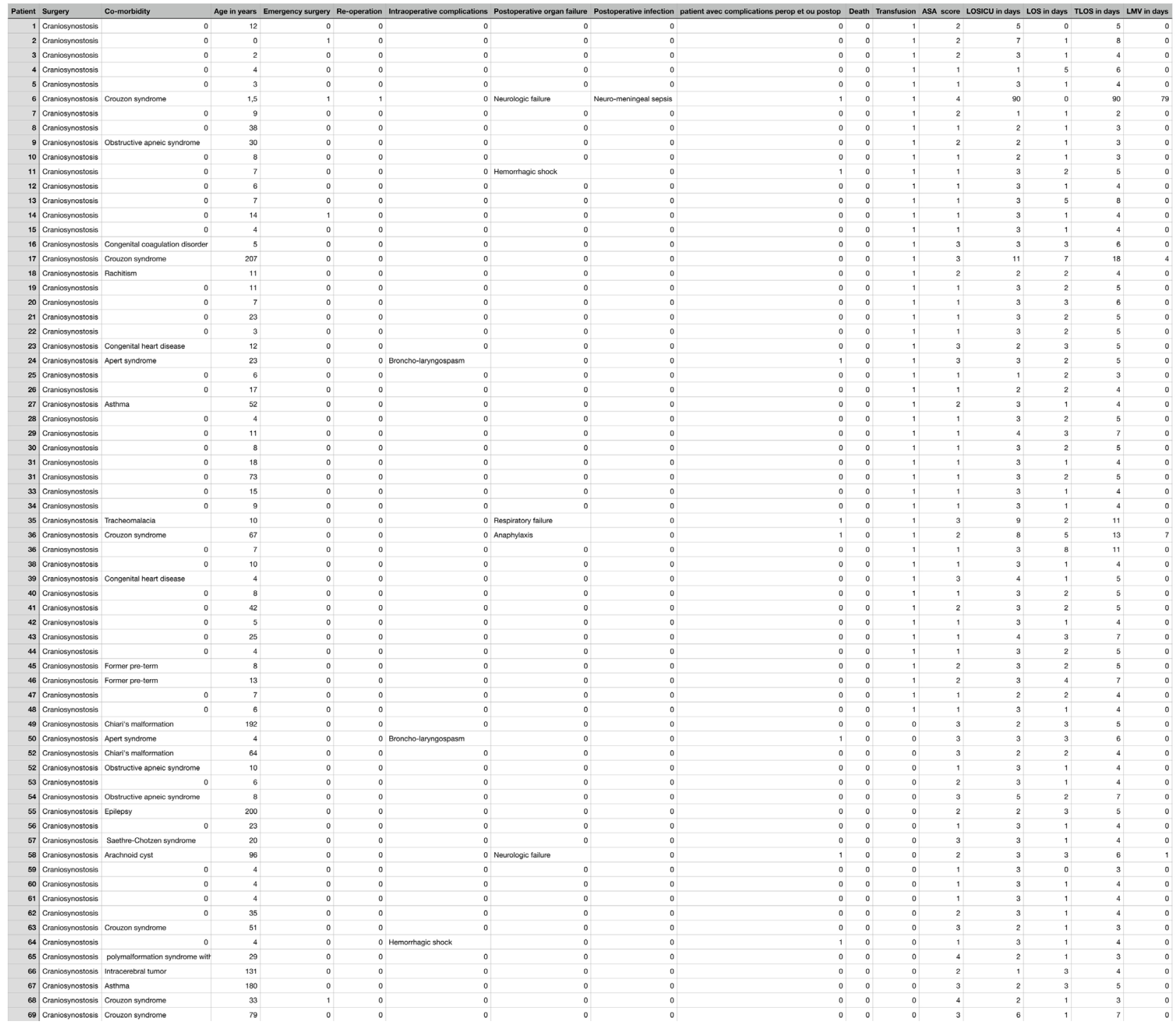

Figure S1. Clinical characteristics. 